\title{
NFIRAOS: TMT's facility adaptive optics system
}

Glen Herriot, David Andersen, Jennifer Atwood, Corinne Boyer, Annie Beauvillier, et al.

Glen Herriot, David Andersen, Jennifer Atwood, Corinne Boyer, Annie Beauvillier, Peter Byrnes, Rod Conan, Brent Ellerbroek, Joeleff Fitzsimmons, Luc Gilles, Paul Hickson, Alexis Hill, Kate Jackson, Olivier Lardière, John Pazder, Thomas Pfrommer, Vlad Reshetov, Scott Roberts, Jean-Pierre Véran, Lianqi Wang, Ivan Wevers, "NFIRAOS: TMT's facility adaptive optics system," Proc. SPIE 7736, Adaptive Optics Systems II, 77360B (12 July 2010); doi: 10.1117/12.857662

SPIE Event: SPIE Astronomical Telescopes + Instrumentation, 2010, San Diego, California, United States 


\title{
NFIRAOS - TMT's facility adaptive optics system
}

Glen Herriot ${ }^{\mathrm{a}}$, David Andersen ${ }^{\mathrm{a}}$, Jennifer Atwood ${ }^{\mathrm{a}}$, Corinne Boyer ${ }^{\mathrm{b}}$, Annie Beauvillier ${ }^{\mathrm{a}}$, Peter Byrnes $^{\mathrm{a}}$, Rod Conan ${ }^{\mathrm{d}}$, Brent Ellerbroek ${ }^{\mathrm{b}}$, Joeleff Fitzsimmons ${ }^{\mathrm{a}}$, Luc Gilles ${ }^{\mathrm{b}}$, Paul Hickson ${ }^{\mathrm{c}}$, Alexis Hill ${ }^{\mathrm{a}}$, Kate Jackson ${ }^{\mathrm{d}}$, Olivier Lardière ${ }^{\mathrm{d}}$, John Pazder ${ }^{\mathrm{a}}$,Thomas Pfrommer ${ }^{\mathrm{c}}$, Vlad Reshetov ${ }^{\mathrm{a}}$, Scott Roberts $^{\mathrm{a}}$, Jean-Pierre Véran ${ }^{\mathrm{a}}$, Lianqi Wang ${ }^{\mathrm{b}}$, Ivan Wevers ${ }^{\mathrm{a}}$

${ }^{a}$ Herzberg Institute of Astrophysics, 5071 W. Saanich Rd., Victoria, Canada V9E 2E7;

${ }^{\mathrm{b}}$ TMT Observatory Corp., 2632 E. Washington Blvd., Pasadena, CA 91107;

${ }^{c}$ Dept. of Physics \& Astronomy, University of British Columbia, 6224 Agricultural Rd., Vancouver, Canada, V6T 1Z1

${ }^{\mathrm{d}}$ Adaptive Optics Lab, ELW A212, University of Victoria, Victoria, Canada, V8W 3P6.

\begin{abstract}
NFIRAOS, the TMT Observatory's initial facility AO system is a multi-conjugate AO system feeding science light from 0.8 to 2.5 microns wavelength to several near-IR client instruments. NFIRAOS has two deformable mirrors optically conjugated to 0 and $11.2 \mathrm{~km}$, and will correct atmospheric turbulence with 50 per cent sky coverage at the galactic pole. An important requirement is to have very low background: the plan is to cool the optics; and one DM is on a tip/tilt stage to reduce surface count. NFIRAOS' real time control uses multiple sodium laser wavefront sensors and up to three IR natural guide star tip/tilt and/or tip/tilt/focus sensors located within each client instrument. Extremely large telescopes are sensitive to errors due to the variability of the sodium layer. To reduce this sensitivity, NFIRAOS uses innovative algorithms coupled with Truth wavefront sensors to monitor a natural star at low bandwidth. It also includes an IR acquisition camera, and a high speed NGS WFS for operation without lasers. For calibration, NFIRAOS includes simulators of both natural stars at infinity and laser guide stars at varying range distance. Because astrometry is an important science programme for NFIRAOS, there is a precision pinhole mask deployable at the input focal plane. This mask is illuminated by a science wavelength and flat-field calibrator that shines light into NFIRAOS' entrance window. We report on recent effort especially including trade studies to reduce field distortion in the science path and to reduce cost and complexity.
\end{abstract}

Keywords: Thirty Meter Telescope, TMT, NFIRAOS, Adaptive Optics

\section{INTRODUCTION}

Several of the early-light suite of instruments for the Thirty Meter Telescope (TMT) will be fed by a facility adaptive optics system, NFIRAOS (Narrow Field Infrared Adaptive Optics System) that compensates atmospheric turbulence. The initial version of NFIRAOS will have diffraction-limited performance in the near IR with 50 per cent sky coverage at the galactic pole. This capability will be achieved via an order 60x60 multi-conjugate AO system (NFIRAOS) with two deformable mirrors optically conjugate to ranges of 0 and $11.2 \mathrm{~km}$, six high-order wavefront sensors observing laser guide stars in the mesospheric sodium layer, and several low-order, infrared natural guide star wavefront sensors located within each client instrument. Among these on-instrument sensors, there will be at least one tip/tilt/focus sensor, and most instruments will also have an additional pair of tip/tilt sensors.

As seen in Figure 1 NFIRAOS resides on one of the two Nasmyth platforms of the TMT. The input beam from the telescope arrives from the tertiary mirror, visible in the center of the main 30-m mirror. NFIRAOS corrects the science light and supports up to three client instruments shown here as cylinders on the top, bottom and the side in the right half of Figure 1. The dark grey exoskeleton carries a total of $47 \mathrm{~T}$ for NFIRAOS, three instruments and a science calibration light source. The cooled electronics enclosure also sits on the Nasmyth platform.

The left half of Figure 1 shows the early light instrument complement, with NFIRAOS standing on a support structure from the Nasmyth platform 7 meters below. This support structure is provided by the telescope and carries the exoskele-

Adaptive Optics Systems II, edited by Brent L. Ellerbroek, Michael Hart, Norbert Hubin, Peter L. Wizinowich, Proc. of SPIE Vol. 7736, 77360B - @ 2010 SPIE · CCC code: 0277-786X/10/\$18 - doi: 10.1117/12.857662 
ton of NFIRAOS. The support structure is intended to be temporary for the lifetime of NFIRAOS, and may in due course be reconfigured to accommodate NFIRAOS' eventual successors.

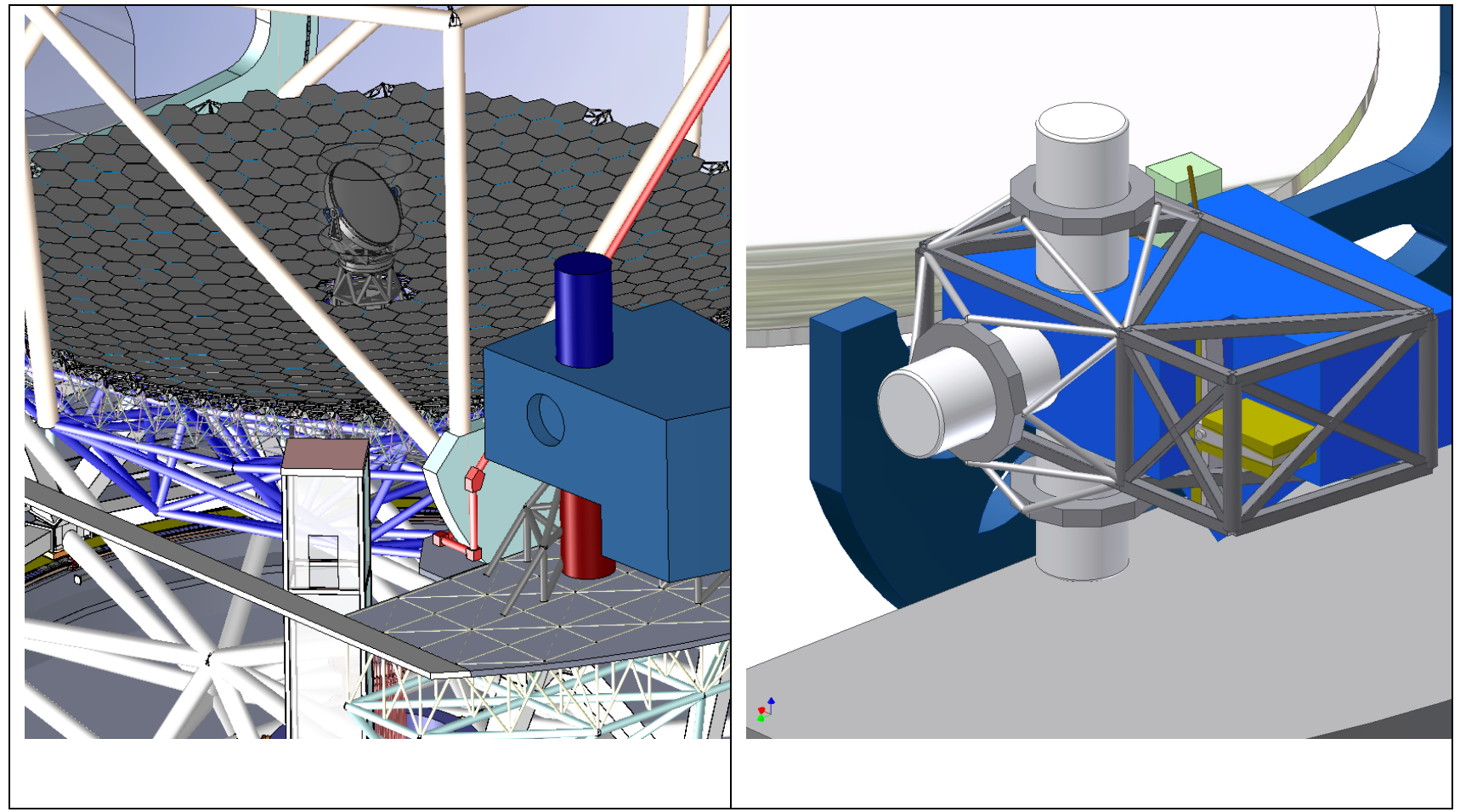

Figure 1 NFIRAOS on Nasmyth Platform of TMT

\section{TOP-LEVEL DESIGN REQUIREMENTS}

The top-level requirements documents for the TMT observatory specify that the facility AO system shall have:

- 85 per cent throughput from 0.8 to $2.5 \mu \mathrm{m}$

- Thermal emission $<15$ per cent of background from sky and telescope

- $187 \mathrm{~nm}$ RMS wavefront error on-axis, and tilt-removed WFE of $190 \mathrm{~nm}$ on a 30" field of view (FoV)

- High enclosed energy within 160 mas pixels over a 2' FoV

- 50 per cent sky coverage at the Galactic pole

- $2 \%$ differential photometry for a 10 minute exposure on a 30 " FoV

- $50 \mu$ as differential astrometry for a 100 s exposure on a $30 " \mathrm{FoV}$

- Error falling as $\mathrm{t}^{-1 / 2}$ to a systematic floor of 10 mas

- System available from standby within 10 minutes

- 5 minutes to acquire a new field

- $\quad<1$ per cent unscheduled downtime 


\section{NFIRAOS ARCHITECTURE}

To meet the above requirements, we have derived a system concept with the following features.

In order to achieve high image quality uniformly over the field of view in the near infrared, NIFROAS employs very high order (60x60) wavefront sensing and correction. NIFIRAOS is a multi-conjugate adaptive optics (MCAO) system whose dual deformable mirrors correct a wide field of view. To control these mirrors NFIRAOS uses tomographic wavefront reconstruction with multiple (6) guidestars.

The $50 \%$ sky coverage requirement, at the galactic pole is in conjunction with simultaneously meeting the specification on wavefront error. NFIRAOS will use six laser guide stars and near-infrared tip/tilt and focus sensing. The tip/tilt sensors are within instruments and use infrared stars sharpened by the adaptive optics system, so their diffraction limited image cores can be sensed accurately with better signal to noise. As well, red stars are more numerous and less obscured by dust. We expect that the majority of the time, NFIRAOS will be guiding using M stars. The wide 2 arcminute guide field of view corrrected by dual deformable mirrors assists achieving high sky coverage by sharpening guide stars over the full guide field.

Besides the sky coverage benefits, using on-instrument WFSs permits optimizing the pickoff for each science instrument, and reduces differential flexure between the science detector and the tip/tilt and tip/tilt/focus sensors.

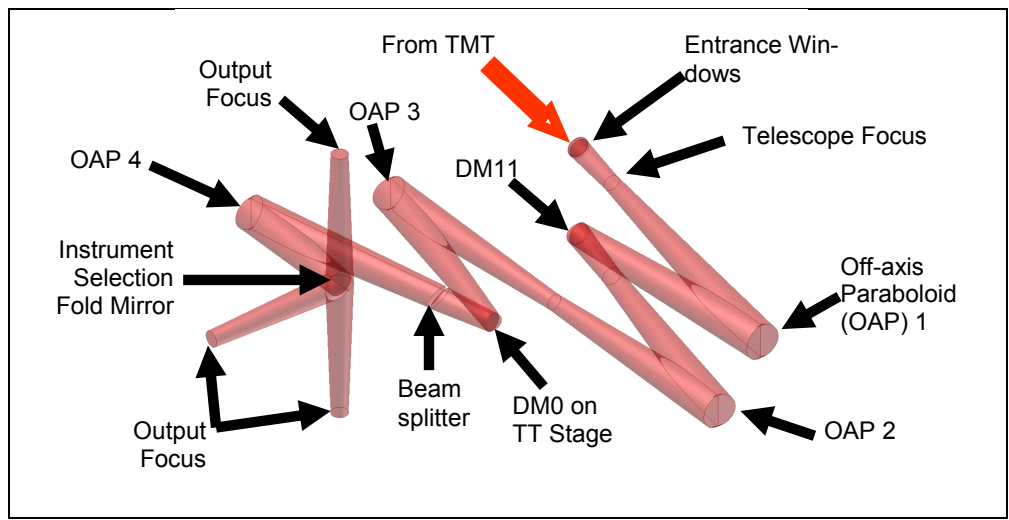

Figure 2 NFIRAOS Science Path

NFIRAOS will also use the three oninstrument WFSs to detect tilt anisoplanatism and instrument rotator errors. Tilt anisoplanatism manifests itself, in a dual-DM multiconjugate AO system, as three modes of image distortion: magnification error, differential magnification in $\mathrm{XY}$ and differential magnification at 45 degrees.

Good optical throughput and low background result from a science path design with a minimum number of surfaces, cooled to minimize emission. Recently to reduce image distortion in the science path to $0.0017 \%$, we have revised the optical design. We have in series, two relays, each with two off-axis paraboloidal mirrors. One DM is located in each relay. As shown in Figure 2 the light from TMT enters via an evacuated dualpane window and is nearly collimated by off-axis paraboloid 1 . The first deformable mirror DM11, is conjugate to 11.2 $\mathrm{km}$ in front of the telescope. The second, DM0, resides on a tip/tilt stage. After DM11, the science light passes through a beamsplitter before being re-imaged by OAP4. Finally an instrument selector fold mirror directs the light to instruments on the top, bottom or side ports.

Figure 3 shows all of the cold NFIRAOS optics from the same viewpoint as the previous figure. The science path is in red. The science beamsplitter diverts visible light downwards from the science path where it is immediately split into a natural visible light path shown in green, and the artificial laser light shown in yellow. This laser light is reimaged by an OAP, second from the left in the figure, and then sent into the laser wavefront sensor trombone optics. These trombone optics are not cooled, and operate at the ambient temperature of the telescope dome. The LGS WFS optics are shown in more detail in Figure 7. 


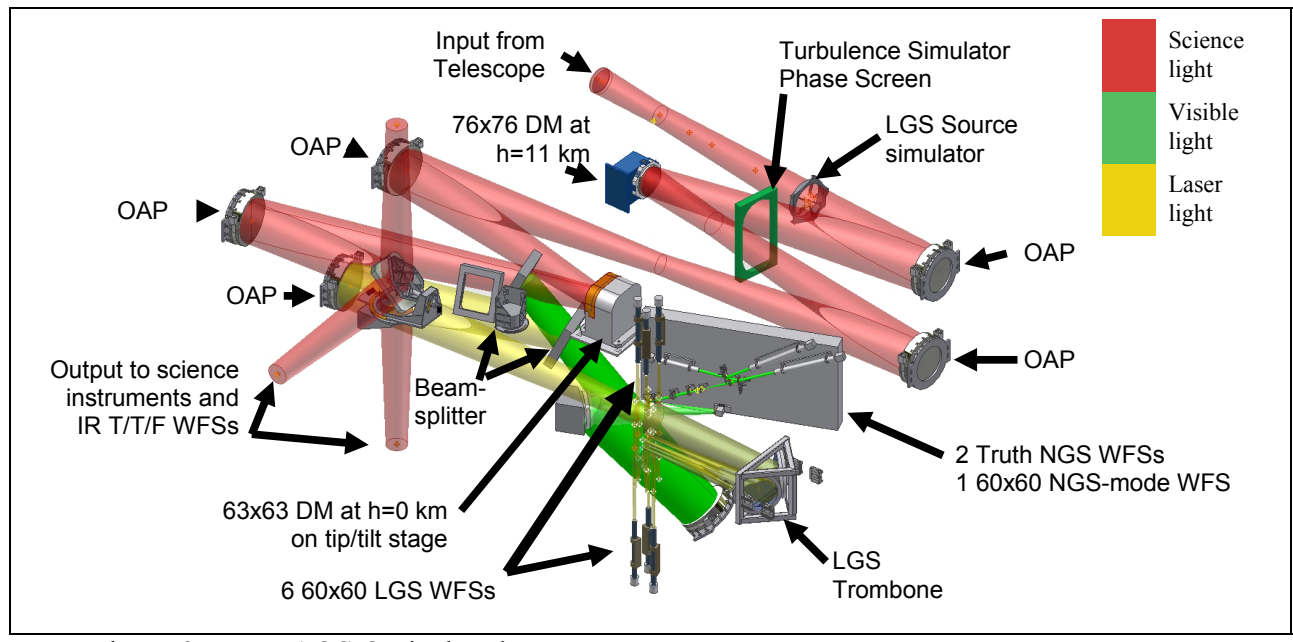

Figure 3 NFIRAOS Optical paths
The natural visible light in green continues downwards through the second beamsplitter and is reflected by a copy of OAP4 that reimages the beam. The reimaged natural visible light is sent to the vertical optical bench containing a collection of visible NGS wave-front sensors described later.

As noted above, NFIRAOS has three copies of OAP4: for reimaging the science, laser and natural visible light. This ap-

proach permits placing beamsplitters in collimated light to reduce aberrations, in particular astigmatism. A block diagram of the optics is shown next.

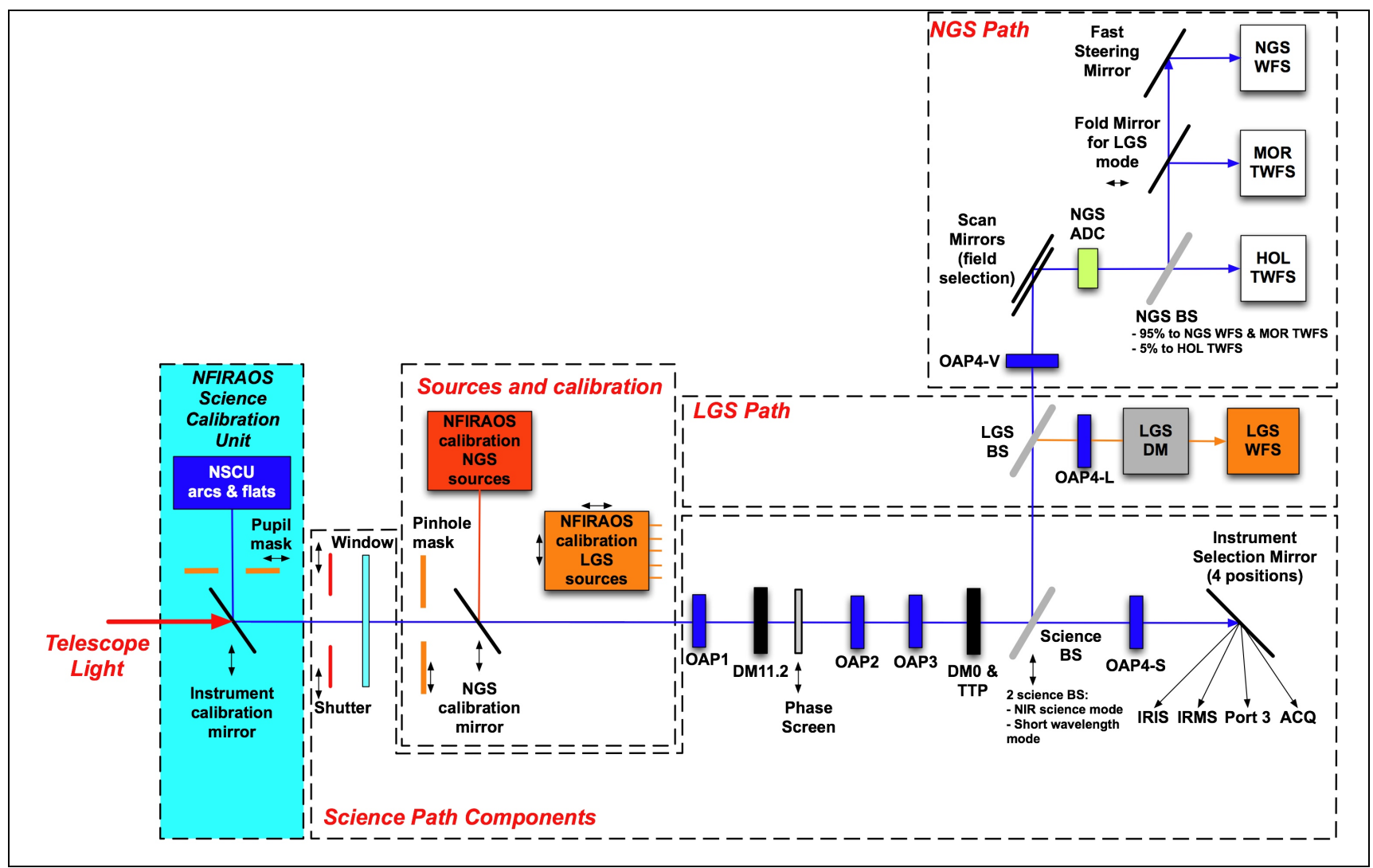

Figure 4 NFIRAOS block diagram of optics

In Figure 4 the telescope light enters from the left, and the science path is shown as the horizontal line from left to right. It passes through the NFIRAOS Science Calibration Unit (NSCU). This is a flat field and wavelength calibrator with a deployable fold mirror that injects calibration light through NFIRAOS to client instruments. This unit also simulates the telescope pupil as a reference for building pupil pointing look-up tables for NFIRAOS as fed by TMT. The instrument calibration mirror is normally retracted during observing and the light passes from the telescope directly through a shut- 
ter and through the dual pane window. This fused-silica window is evacuated to reduce heat leakage from the dome into NFIRAOS, and to reduce local seeing degradation.

Inside the window, are a deployable pinhole mask and fold mirror that direct simulated asterisms of natural guide stars into NFIRAOS for daytime calibration. A deployable array of LGS sources supported by spiders is next. Continuing to the right, the beam passes OAP1 to OAP3 and the two DMs. It goes through the science beamsplitter before being collimated by OAP4-S and then directed to one of three instruments or the acquisition camera (ACQ).

Visible light is reflected by the beamsplitter and travels upward in this diagram to a second beamsplitter. Laser light reflects from this LGS beamsplitter, is reimaged by OAP4-L, and passes through the zoom optics to the laser wavefront sensors. These optics include a common trombone for all 6 laser stars, followed by six magnetic DMs for non-common path compensation of each one individually. Natural visible light passes through the LGS beamsplitter and is reimaged by OAP4-V. A pair of scan mirrors selects a single natural star and directs it through an atmospheric dispersion compensator (ADC). In normal laser guide star operation, a beamsplitter divides the natural guide starlight between a pair of low-speed truth wavefront sensors (TWFs). These TWFSs establish offsets to be applied to the laser guide star wavefront sensors to compensate for residual non-common path aberrations and to track offsets caused by changes in seeing and the sodium layer.

The HOL TWFS is a high-order, low-bandwidth 120x120 with oversampled pixels to reduce aliasing. It operates with integration times of about a minute. The MOR TWFS is a $12 \times 12$ wavefront sensor with $0.3 \mathrm{~Hz}$ integration time.

Additionally, for operation without lasers on bright natural stars, the visible light will be measured by a $60 \times 60$ NGS WFS at high frame rates, while $5 \%$ of the light continues to the TWFS. To track gain changes on centroiding the quad cells of the NGS wavefront sensor caused by seeing variations, a fast steering mirror introduces 50 mas tip/tilt dither on the sky.

An isometric view showing all of the optics on their space frame support is in igure 5. Here the light from the telescope enters from the top and passes all the way through to the right hand side of the diagram where it encounters the first optical surface, an OAP. Eventually the instrument selection fold mirror sends the science light up, down or left to the science ports. Alternatively it goes to the infrared acquisition camera (not shown).

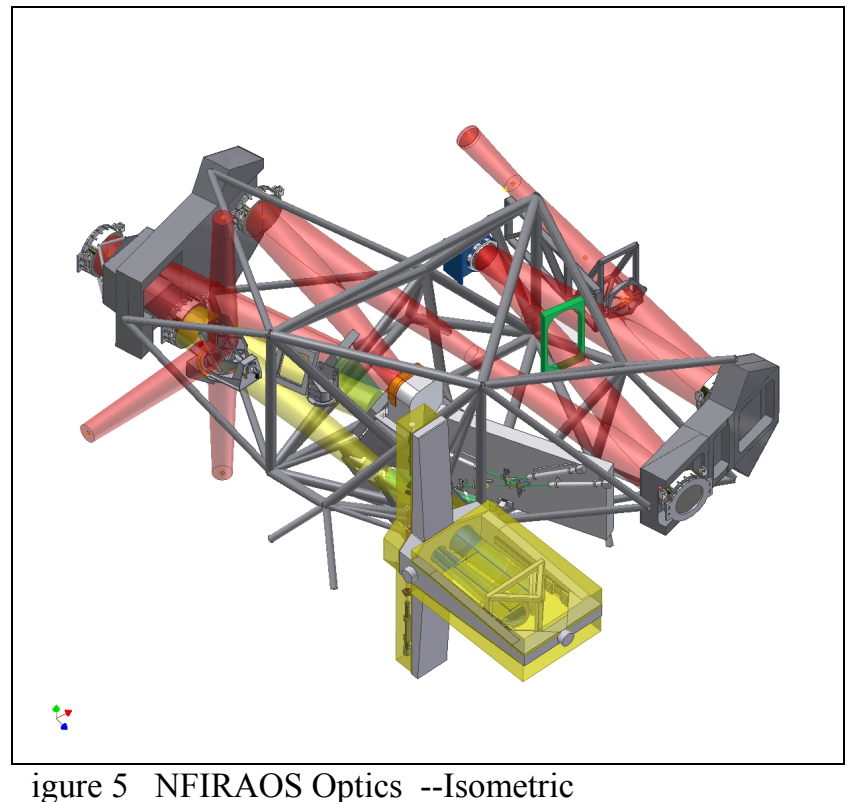


An overall view of the exterior of NFIRAOS is depicted in Figure 6. NFIRAOS is rotated 90 degrees counterclockwise from the previous figure. The light from the telescope passes through the opening in the Science Calibration Unit (NSCU) shown in green on the left. The three science instruments are the grey cylinders on the top and bottom and side. Instruments incorporate rotator bearings for field-derotation. The bearings are at the center of gravity near the midpoint of each instrument. The dark gray exoskeleton, is the instrument support structure (ISS) that gathers the loads from the three instruments, the NSCU and NFIRAOS' optical bench and transmits the load to the telescope structure. The blue box is the cooled enclosure for the science optics. The LGS zoom optics are in the yellow box at the right of this view.

\subsection{Laser WFS Zoom Optics}

Figure 7 shows the laser wavefront sensor zoom optomechanics. The asterism of laser guide stars is in the shape of a pentagon with a sixth star in the middle of the field. The six guide stars come at a distance that varies

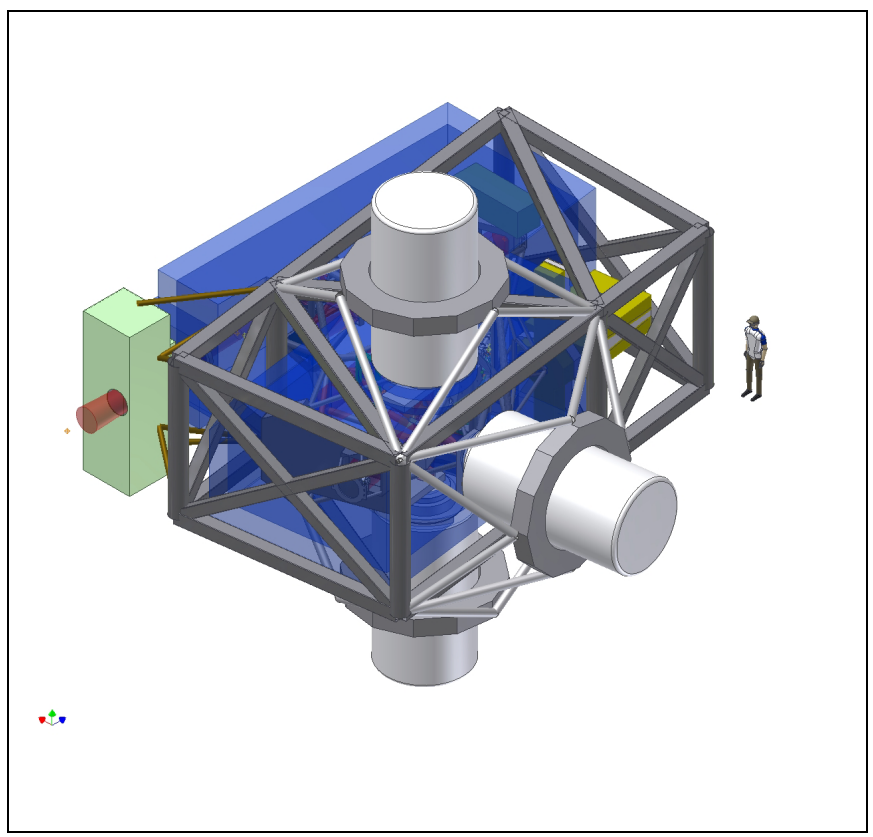

Figure 6 NFIRAOS with three instruments and NSCU with the zenith angle of the telescope and the height of the sodium layer. The zoom optics have three main roles: (i) to refocus on the sodium beacons at range distances from 85 to $235 \mathrm{~km}$; (ii) to accurately image the actuator grid of DM0 onto the lenslet arrays in the LGS WFSs; and (iii) to compensate for the aberrations induced by the telescope and science optics.

These latter optics are designed for objects at infinity, and when imaging laser beacons as close as $85 \mathrm{~km}$, then the telescope causes field-dependent aberrations, which the trombone optics must accurately compensate. The as-built allowance for residual wavefront error in the laser WFS optics is $<150 \mathrm{~nm}$ RMS for all range distances. We expect that software calibration of non-common path errors can attenuate this by a factor of 5 .

At the right of Figure 7 is the trombone. After that the six guide stars come to focus, at a fixed focal plane. Note that the laser launch system rotates the asterism of laser beacons on the sky to keep it aligned with these WFSs in NFIRAOS as the telescope tracks. Each star is picked off by fold mirror and sent through a field stops and is collimated onto a magnetic deformable mirror. These deformable mirrors are programmed to replicate the expected non-common path aberrations vs. sodium range distance, caused by the telescope and NFIRAOS science optics. The magnetic DMs will be calibrated in the daytime by deployable fibres at the field stops, and adjusted until the measurements from the LGS WFS match expectations from ray tracing. The final tweak of non-common path calibration will be by processing science instrument images and adding small electronic offsets to the slopes measured from the WFSs.

After the magnetic DMs, the light travels to the six WFS barrels. Each order 60x60 WFS barrel is equipped with a polar coordinate CCD with 204792 pixels resulting in 5792 slope

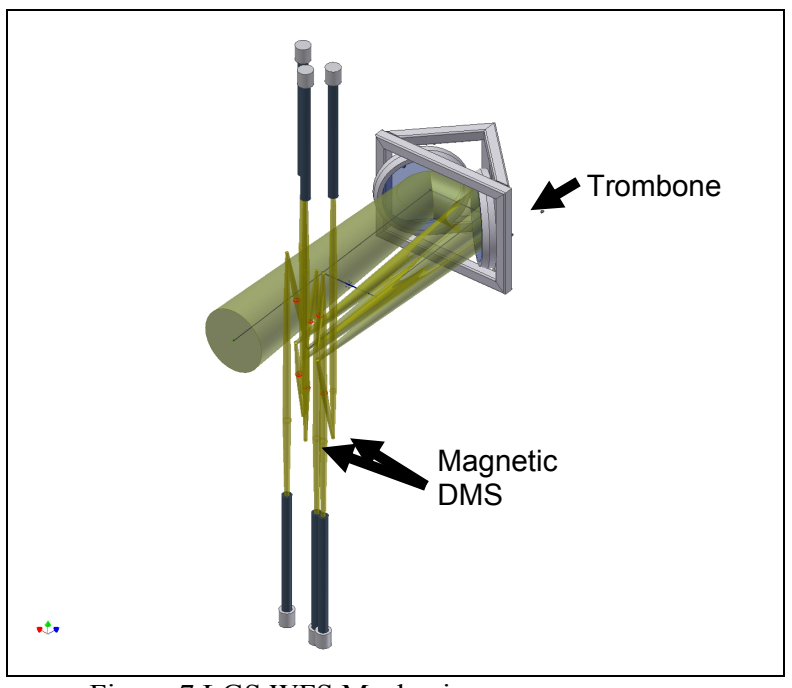

Figure 7 LGS WFS Mechanics measurements per WFS. 


\subsection{Instrument Mounting}

Instruments are responsible for field derotation as well as tip/tilt sensors and atmospheric dispersion compensators. The derotator bearing, cable wraps and rotary seals are provided by the instruments. To exchange an instrument, NFIRAOS closes gate valves to keep the cold air inside. Unused port holes are filled with manually-installed insulated plugs.

As depicted in Figure 6, the instruments attach via trusses (light gray in the figure). Each interface is identical with the exception of the truss for the side instrument, which would be replaced to move the side instrument to a top or bottom port. This difference results from unavoidable triangulation differences on the top and side of NFIRAOS' instrument support structure. Sig-

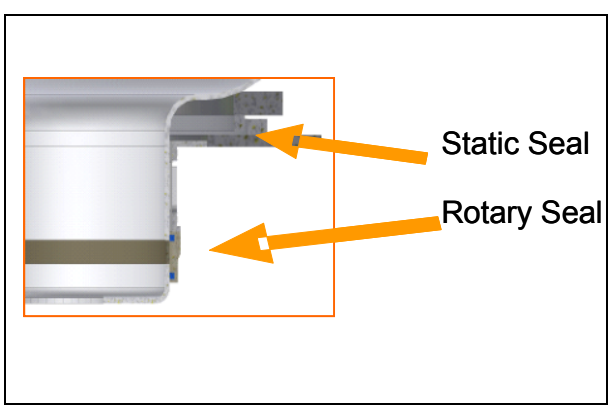

Figure 8 Instrument Mounting Seals nificant effort has been put onto finite element analysis of this structure to assess thermal performance, vibration sensitivity, and earthquake survivability.

In a typical earthquake analysis we represent the mass and moments of individual optical mounts or client instruments by tripods. Slender posts within each tripod allow us to interrogate the vertex motion of each optic. To excite this FEA model, we drive it with time series of earthquake accelerations as transmitted through the telescope and Nasmyth platform structure to NFIRAOS. The upper instrument experiences 5 Gs. Accelerating the mass moment of inertia of the upper instrument, whose center of gravity is located two meters above the upper mounting face creates the greatest stress on the instrument support structure. Thus the current baseline has instruments supported by a truss, rather than by their mating face on NFIRAOS.

\subsection{Cooling NFIRAOS Optics}

To reduce background, NFIRAOS optics are cooled to $-30 \mathrm{C}$. The initial cool down is done by air handling units, which are switched off once NFIRAOS is cold. In operation, heat is intercepted by buried cold plates in the insulated walls. Previously we planned to use vacuum insulated panels, but with the increase in size to enclose the low-distortion 4-OAP optical design, and the elaborate non-overlapping tiling in the panels to prevent heat leaks this became unaffordable. Recently, we have switched to polystyrene foam insulation. This change has been enabled by increasing the thickness of the buried cold plate and selecting the aluminum alloy for increased conductivity.

Less than a kilowatt is extracted from the dome and is intercepted by the embedded cold plates. These are edge-cooled by R-507 refrigerant in fixed pipes, allowing removal of panels without disconnecting plumbing. Heat flow within the cold plate causes several degrees of temperature variation across the panels.

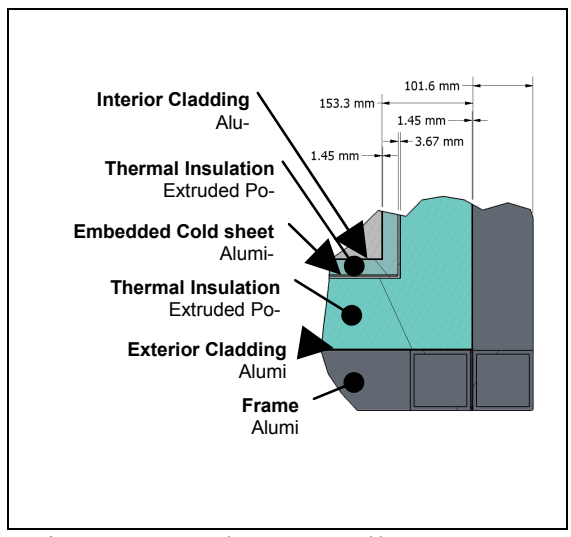

Figure 9 Enclosure Walls The interior layer of thermal insulation and the interior cladding serve to maintain the uniformity of the interior walls within $0.5 \mathrm{C}$, from center to edge of each panel. 


\section{ERROR BUDGET}

The error budget shown in Figure 10 is constructed for onaxis objects and for $50 \%$ sky coverage at the Galactic pole. This means there is $50 \%$ probability of achieving this level of tip/tilt performance for a random field near the pole. At lower latitudes of course, the sky coverage will be greater.

The top-level wavefront error of $187 \mathrm{~nm}$ results in Strehls of $0.41,0.60$, \& 0.75 in $\mathrm{J}, \mathrm{H}$, and $\mathrm{K}$ band.

This budget applies to median seeing, median sodium column density, and $75^{\text {th }}$ percentile high wind windshake of the telescope. The budget includes errors of the telescope, instrument, and second order effects such as DM hysteresis.

The error increases by 50-70 nm RMS for 30" FoV (sitedependent).

NFIRAOS is also expected to provide a factor of three improvement in enclosed energy within a 160 mas slit for the IRMS spectrograph at the edge of a 2' FoV

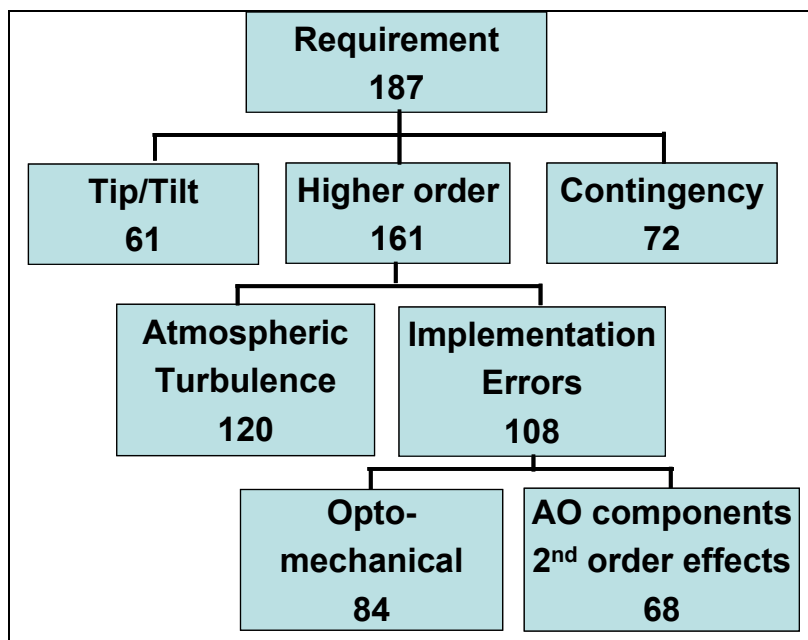

Figure 10 Top level error budget nm RMS

\section{CONCLUSION}

To summarize, updating the Preliminary Design of NFIRAOS is underway, with overall layouts and opto-mechanical designs well advanced. We have completed detailed performance estimates, supported by component prototyping and lab tests.

We have revised the science path to reduce distortion, and developed new, cost-effective designs for the calibration sources, turbulence simulator, insulation, and laser WFS optics.

\section{ACKNOWLEDGEMENTS}

The authors gratefully acknowledge the support of the TMT partner institutions. They are the Association of Canadian Universities for Research in Astronomy (ACURA), the California Institute of Technology and the University of California. This work was supported as well by the Gordon and Betty Moore Foundation, the Canada Foundation for Innovation, the Ontario Ministry of Research and Innovation, the National Research Council of Canada, the Natural Sciences and Engineering Research Council of Canada, the British Columbia Knowledge Development Fund, the Association of Universities for Research in Astronomy (AURA) and the U.S. National Science Foundation.

\section{REFERENCES}

[1] G. Sanders and J. Nelson, “The status of the Thirty Meter Telescope project”, Proc. SPIE 7733-69 (2010).

[2] B. E. Ellerbroek, , et al., "First light adaptive optics systems and components for the Thirty Meter Telescope," Proc. SPIE 7736 (2010).

[3] J.-P. Véran, C. Irvin, and G. Herriot, "Implementation of type-II tip-tilt control in NFIRAOS, with woofer-tweeter and vibration cancellation," Proc. SPIE 7736-167 (2010).

[4] R. Conan, J.-P. Véran, and K. J. Jackson, "Experimental validation of type-II tip-tilt control in a woofer-tweeter adaptive optics system," Proc. SPIE 7736-169 (2010).

[5] D.-S. Moon, " The Science Calibration System for the TMT NFIRAOS and Client Instruments: Requirements and Design Studies," Proc. SPIE 7736, (2010). 